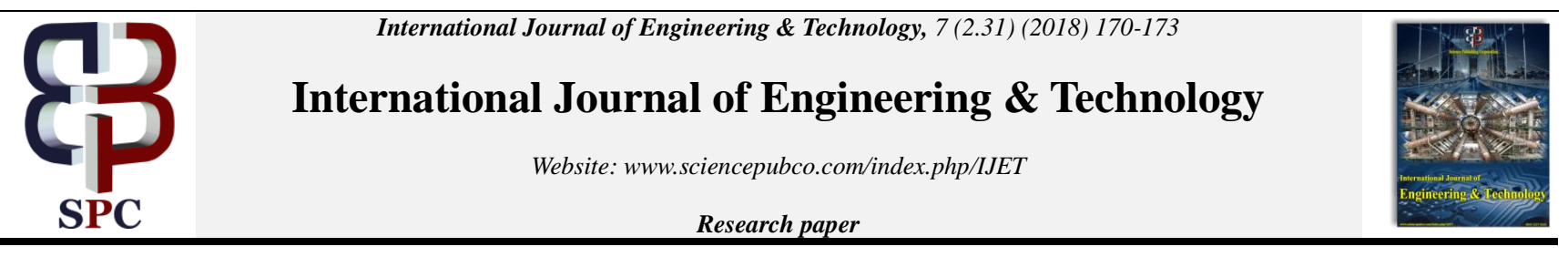

\title{
Food grain storage management system
}

\author{
A. Akila ${ }^{*}$, P. Shalini ${ }^{2}$ \\ ${ }^{1}$ Department of Computer Science, Vels Institute of Science Technology and Advanced Studies(VISTAS), Chennai, India. \\ ${ }^{2}$ Department of MBA, Vels Institute of Science Technology and Advanced Studies(VISTAS), Chennai, India. \\ *Corresponding authorE-mail:akila.scs@velsuniv.ac.in
}

\begin{abstract}
Food grain Wastage cripples a country's economy to a great extent. Food grain wastage is also associated with wastage of water, manpower during agricultural activities and electricity power used in food processing industries. It even causes deforestation. Adequate measures have to be taken to properly store the food grains so that they remain edible. The proposed storage management system uses the sensors to measure the levels of humidity, temperature and ammonia gas which will help us monitor quality of the food grains. The main idea is to identify the quality of the food grains using the sensors such as Temperature, Humidity and Ammonia Gas. The Quality of the food grain is measured using the factors like Humidity, Temperature and Ammonia gas sensors and sent through Wireless Communication to the server and the server makes the decision and alarms about the quality of the food grain to the maintenance people. Food grain Wastage cripples a country's economy to a great extent. Food grain wastage is also associated with wastage of water, manpower during agricultural activities and electricity power used in food processing industries. It even causes deforestation. Adequate measures have to be taken to properly store the food grains so that they remain edible. The proposed storage management system uses the sensors to measure the levels of humidity, temperature and ammonia gas which will help us monitor quality of the food grains. The standard of identifying the food quality could be improved by using more sensors and can be implemented in the Food Storage Industry.
\end{abstract}

Keywords: Food grain quality control, humidity sensor, temperature sensor, ammonia gas sensor.

\section{Introduction}

Food grain wastage is an important issue that needs to be addressed soon in India. According to the United Nations development program, up to $40 \%$ of the food produced in India is wasted.

Food grain wastage cripples a country's economy to an extent that most of us are unaware. When food grains are wasted, there is wastage of water and manpower which is used during cultivating the food grains. There is also a lot electric power wasted during the processing of food grains. It also leads to deforestation.

This paper presents an automated system that senses the quality of the food grains and constantly informs the administrator about how fresh the food grains are. The physical factors such as humidity, temperature and ammonia gas are taken into consideration to assess the quality of the food grains. The section II gives the overview and literature study of various work done to control the wastage of food grains. The section III gives the framework of the circuit designed to control food grain spoilage and the last section concludes with the benefits of the proposed system.

\section{Literature study}

The food grains have to be stored till the next harvest because it is the main economy of the nation. The grains could be infected by insects and fungus. The major problem of food security in Indian agriculture is inadequate infrastructure and lacks an efficient supply chain management. India is one among the largest producer of food grains. Agricultural products contribute to about 13.8 percent of country's gross domestic product. On the contrary India is one of the countries which has large amount of food scarcity problem. The paper by Sazzad Parwez discusses two supply chain management models to streamline supply chain in India[1]. The first model is ITC E-Choupal supply chain model[1]. With E-Choupal, farmers are constantly updated about market price (worldwide), the demand for the grain, etc. This is done with the help of Kiosk. The information is gathered from various sources like the department of Agriculture, Government Universities, Indian Meteorological Department, Input Firms, and retailers. The weather information helps the farmers to take care of the crops accordingly. E-Choupal is being successfully used in more than 38000 villages through 6500 kiosks across various states in India.

Another supply chain model is Reliance Fresh supply chain framework which gets information from farmers and city processing centers. Collection centers works as intermediate to collect the food grains and fulfill the demand of the public through retail outlets. The centers provide a price band to the food grains which helps the farmers gain maximum profitability. The food grains have to be protected from the natural degrading factors like humidity, temperature. The infrastructure of the storage area plays a vital role in maintaining the quality. The two models mentioned provide better infrastructure to maintain the quality of the food products [1].

Groundnuts are used for producing oil and are sometimes directly consumed[2]. There was a study carried out by G.Harish to prevent spoilage of groundnuts. The groundnuts are affected by the pest such as bruchid beetle if is stored for a long term. Aluminium phosphate is usually used to preserve grains but is hazardous to health as it causes serious toxic effect to lungs, heart and blood vessels. Thus alternative storage options were exploited. Many storage bags like the lino bag, gunny bag and fertilizer bag were used to preserve grains. The study concluded that the super grain bag showed better result in winter season by suppressing the C.Serratus insect pest [2]. When stored for long periods under improper conditions food grains are prone to rodent 
attacks and degradation due to other environmental factors. The reason behind rodent attack is lack of hygienic places to store the grain. Apart from rodent attacks variation in temperature, humidity and gas concentration lead to spoilage of food grains.

Models like Partial Least Square (PLS) and Takagi-sugeno (TS) are available to track the quality of the grain and to predict how long the food grains will sustain [3]. The dynamic PLS system with decoupling and dimension reduction represent the multi input multi output system as combination of many single input single output system. With the TS Fuzzy modeling method, the non linear growth process of grain can be controlled effectively. A system was designed having both PLS and TS features which was named as TS-PLS model. The model has reduced the error in prediction and it is effective when compared to the traditional models like PLS and TS models[3].

A real time monitoring and controlling system for grain storage was designed by Vinay Sambhaji and Mahesh S.Kumbhar [4]. The system uses the humidity and temperature sensor to measure the quality of the food grains. The sensors sense the humidity and temperature and send the data to the administrator through Ethernet or wireless devices. The administrator then takes necessary actions according to the received information. The system gives humidity and temperature of the grain which when exceeds the threshold value, the grain starts getting decomposed. The system provides flexibility and the status of the grain in a reliable manner. Any control system developed should be cost effective and have the ability to provide accurate results [4].

The grains can be stored and cooled down using the mechanical ventilators and mechanical vapor compression refrigeration grain chiller. These mechanical devices consume large electric power and thus are not economic. So to overcome such drawbacks, Huilong Luo and his team designed a solar powered Adsorption Chiller [5]. The solar powered adsorption chiller consist of subsystems namely solar water heating unit, silica gel water adsorption chiller, a cooling tower and a fan coil unit.

The solar water heating unit is used to provide hot water to make the adsorption chiller work. The silica gel adsorption chiller has two similar adsorption units and a second stage evaporator with methanol working fluid [5]. The condensers and the adsorbers cool using the cooling tower. The fan coil unit is used to cool the water. The performance of the adsorption chiller is measured using the cooling capacity and the cooling coefficient of the performance. The cooled air from the solar powered adsorption chiller is used in the test grain bins for ventilation. The solar powered adsorption chiller developed by Huilong Luo is a good refrigeration device for low temperature grain storage. Till August 2011 in China, four solar powered adsorption chillers were installed for low temperature grain storage [5].

To detect the present spoilage in stored grains, a carbon dioxide sensor was designed using polyanline boronic acid [6]. The sensor measures the gaseous $\mathrm{CO}_{2}$ levels and the performance of the sensors is evaluated taking in to account factors like temperature and humidity influences. The sensor responded to the variations in $\mathrm{CO}_{2}$ concentration at various levels of humidity and temperature. The performance measures like response time, recovery time, sensitivity, selectivity, stability and response slope were measured at various $\mathrm{CO}_{2}$ levels inside simulated grain bulk conditions. The performance of the polymer $\mathrm{CO} 2$ Sensor has improved after such measures have been taken [6].

The food grains infected by pest and fungus could be controlled by monitoring the temperature, humidity and light [7]. The paper by Santhoshkumar et al discusses about the various sensors used to monitor and control the life of the food grains. The temperature of the grain storage places could be affected by various sources like sun, outside air temperature, heat generated by external respiration of both food and any insect pest in the food. The range of temperature threshold is $14^{\circ} \mathrm{C}$ to $42^{\circ} \mathrm{C}$. The temperature sensor used for this purpose is LM35 [7].

The presence of moisture will help the various insects to live and reproduce. If moisture is controlled, the insects cannot grow. The moisture threshold was set as $9 \%$. The op-amp was used to design the humidity sensor. The insect's preference to light varies. Some insects prefer light and some may not. The threshold value of light was set as 50 to 90 Lux [7].

All these three sensors were connected to Freeduino board and Zigbee. Zigbee [7] is a wireless network technology used with operating in the frequencies $868 \mathrm{MHz}, 902-928 \mathrm{MHz}$ and $2-4 \mathrm{GHz}$. The Zigbee protocol uses Institute of Electrical and Electronics Engineer (IEEE) specification 802.15.4 as the medium access layer and physical layer. The sensors read the value of the attributes such as humidity, temperature and light and compares with the threshold value. If the value is out the threshold range, the information is sent to the receiver side of the network through the microcontroller AT Mega 328 and Zigbee transceiver which is stored in the database for setting the alarm and taking remedial actions to avoid damage to food grains.

In current agriculture scenario, automation plays a vital role. The binding of wireless sensor and mobile system network is needed to control and remotely monitor the environmental changes in a poultry farm. The model will alert the person who monitors the farm about the various environmental parameters like temperature, humidity, etc. by sending message to the registered mobile number. The person can make required action. The level of water is also identified and restricted to a particular level as they play an important role in cooling the poultry farm. The sensor helps to monitor the farm remotely and passing the values to the web with the help of CC3200, which can be later, viewed in Google spreadsheets. The model provides an efficient automated agriculture monitoring system [8].

\section{Proposed model-food storage management system}

\section{Framework of the proposed model}

The proposed model has been designed to control the degrading quality of the food grains. The layout of the server and client framework of the proposed model is given in Figure 1 and Figure 2

Client Side Framework: The Arduino Due R3 Board is used as circuit board to connect the sensors individually. A WIFI Transmitter is also connected. This transmitter is used to transmit the status of each sensor to the server. The framework is shown in Figure 1 .

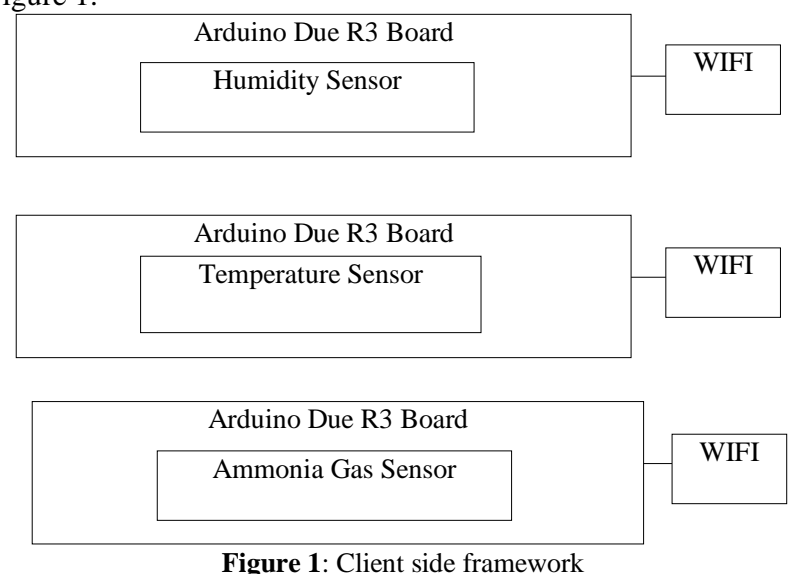

Server side Framework: The server side of the proposed food grain storage management system consists of a WIFI receiver. The receiver receives information about the physical factors that help measure the quality of food grains. The sensor information is then sent to the system. The system then makes the decision about the quality of the grains and triggers the alarm if it the quality is low. The working is shown in Figure 2. 


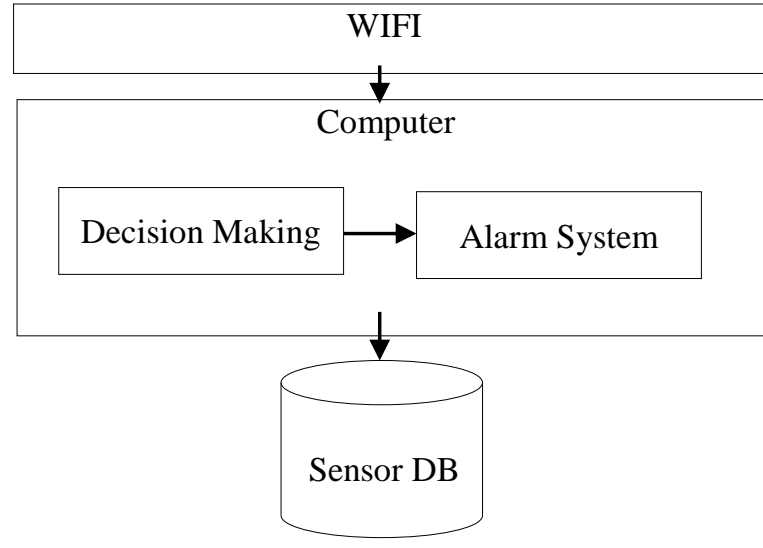

Figure 2: Server side framework

\section{Quality measures}

The quality measures considered in the proposed work are the Humidity, Temperature and ammonia gas.

Humidity: Humidity is the existence of water in air. The quantity of water vapor in air can influence the human ease as well as many manufacturing processes in industries. Humidity also affects the business cost of the product and has impact on the health of the personnel.

The common terms used to indicate the moisture level are absolute humidity, mixing ratio, relative humidity, specific humidity, dew point, frost point, volume ratio and parts per miilon (PPM) by volume. The absolute humidity is the ratio of the mass vapor to volume of gas. The mixing ratio is the ratio of the mass (vapor) to mass (dry gas). The ratio of vapor mass to saturated vapor mass is called the relative humidity. The specific humidity is the ratio of the vapor mass to the total mass. The temperature above $0^{\prime} \mathrm{C}$ at which water vapor in a gas condenses to liquid water is known as the dew point and the temperature at which the water vapor in a gas condenses to ice is frost point. The volume ratio is the ratio of the partial pressure (vapor) to the partial pressure (dry gas). The parts per million by volume is the ratio of the volume $\mathrm{X}$ $10^{6}$ to the volume of dry gas.

Temperature: Temperature in an room can be measured using the Thermometers and Probes. The thermometer is one of the oldest measures and needs certain depth of immersion into the measured medium. Probes uses the resistance elements that changes resistance with temperature which is specified in Equation 1 where $\Delta \mathrm{R}$ is the resistance change, $\Delta \mathrm{T}$ is temperature change and $\mathrm{k}$ is the first order coefficient of resistance of the material .

$\Delta \boldsymbol{R}=\boldsymbol{k} \Delta \boldsymbol{T}$

Ammonia gas: Ammonia gas is a colorless gas which has a odour and it is unsafe to human body. Ammonia gas is an exceeding toxic agent. The deteriorating and decomposing food produces ammonia gas using a variety of micro and macro fungi.

\section{Sensors}

Humidity Sensor: The Humidity sensor could be classified into elastic sensor and electronic sensors. The capacitive and resistive sensors are the two categories of electronic sensors. With the resistive sensor it is difficult to measure the low RH values. Since the variation in impedance is more, it is not easy to manage dynamics and temperature effects. Capacitive sensors on the other hand are linear which can measure RH values from $0 \%$ to $100 \%$ but it can be affected by temperature. As capacitive sensors have low temperature effect, they are often used over wide temperature ranges without active temperature compensation. The capacitive sensors require complex circuit and also needs regular calibration. The humidity sensor should be selected depending on accuracy, linearity, hysteresis, calibration errors and long term stability.
HS1101 Humidity capacitive sensor is selected as Humidity sensor for the proposed model.

Temperature Sensor: The resistance temperature detector which uses specialized pure metal detectors are highly accurate and stable for a long period of time. Transcat 7010T resistance temperature detector (RTD) is used in the proposed model.

Ammonia gas Sensor: There are different types of sensors used by sensor devices to detect ammonia content. A few commonly used sensors are polymer based ammonia sensors, Metal oxide based sensors, catalytic ammonia sensors and optimal ammonia sensors as ammonia sensors. The sensitivity of the polymer based sensors decrease overtime on continual exposure to ammonia. When Metal oxide sensors are exposed to ammonia, the interface between the ammonia and oxygen causes in oxidation of the gas at the surface which later causes removal of oxygen from the grain surface. The metal oxide, catalytic and optimal ammonia sensors take a long time to respond. So to overcome disadvantages associated with existing sensors, a tunable electronic material with pores in oxide on silicon (TEMPOS) is used. MQ135 probe hazardous gas ammonia sulfide detection sensor is selected as ammonia sensor in the proposed food management system.

\section{Algorithm for sensor alarming}

Step 1: Read the Humidity, Temperature and Ammonia Gas value using the sensor

Step 2: If the Humidity value is less than humidity threshold, generate an alarm voice specifying to take care of Humidity of the room

Step 3: If the Temperature in less than or higher than the normal temperature threshold range, an alarm specifying the caution about the room temperature is generated.

Step 4: If the ammonia gas value available is below the ammonia threshold, an alarm voice specifying the ammonia gas value of the room is generated.

Future Enhancement: The number of Humidity, Temperature and Ammonia Gas Sensors should be increased if the size of the room where the food grain is stored could not be sensed by a single Humidity, Temperature and ammonia gas sensors. The administrator should be informed about the sensor id so that the administrator can take necessary actions in that area of the room.

\section{Conclusion}

The prototype of the client and server side proposed model is designed. Humidity, temperature and ammonia gas are used as quality measures for the detection of the quality of food grain in the proposed model. The implementation of the system has to be carried out to prove the real potential of the proposed food grain storage management system.

\section{References}

[1] Parwez S, "Food Supply Chain Management in Indian Agriculture: Issues, Opportunities and Further Research", African Journal of Business Management, Vol.6, No.14, (2014), pp.572-581, 2014.

[2] Harish G, Nataraja MV, Ajay BC, Holajjer P, Savaliya SD \& Gedia MV, "Comparative efficacy of storage bags, storability and damage potential of bruchid beetle", Journal of food science and technology, Vol.51, No.12, (2014), pp.4047-4053.

[3] Wu L, Hui Y \& Li M, "The Storage Grain and Environment Modeling Based on TS-PLS", International Journal of Smart Home, Vol.10, No.1, (2016), pp.23-30.

[4] Suryawanshi VS \& Kumbhar MS, "Real Time Monitoring \& Controlling System for Food Grain Storage", International Journal of Innovative Research in Science, Engineering and Technology, Vol.3, (2014), pp.734-738.

[5] Luo H, Wang R, Dai Y \& Shen J, "Low-temperature grain storage with a solar-powered adsorption chiller: a case study", International journal of green energy, Vol.11, No.1, (2014), pp.5059. 
[6] Neethirajan S, Freund MS, Jayas DS, Shafai C, Thomson DJ \& White NDG, "Development of carbon dioxide (CO2) sensor for grain quality monitoring", biosystems engineering, Vol.106, No.4 (2010), pp.395-404.

[7] Kumar S, Hiremath V \& Rakhee K, "Smart sensor network system based on ZigBee technology to monitor grain depot", International Journal of Computer Applications, Vol.50, No.21, (2012), pp.3236.

[8] Goud KS \& Sudharson A, "Internet based Smart Poultry Farm", Indian Journal of Science and Technology, Vol.8, No.19, (2015), pp.1-5. 